\title{
Mitral annular kinetics in mitral regurgitation
}

\author{
Xiaoxia Zhang ${ }^{1,2^{*}}$, Chun G Schiros ${ }^{3}$, Mustafa I Ahmed ${ }^{3}$, David S McGiffin ${ }^{3}$, Steven G Lloyd ${ }^{3,4}$, Louis J Dell'Italia, ${ }^{3,4}$, \\ Thomas S Denney ${ }^{1,2}$, Himanshu Gupta ${ }^{3,4}$
}

From 17th Annual SCMR Scientific Sessions

New Orleans, LA, USA. 16-19 January 2014

\section{Background}

Mitral annular (MA) kinetics during the cardiac cycle influences both systolic and diastolic function of the LV. Here we describe a novel cMRI approach to evaluate mitral annular kinetics and its relationship in mitral regurgitation post surgical repair.

\section{Methods}

Population: Patients with degenerative mitral regurgitation $(\mathrm{n}=35)$ with normal LV EF pre and 12 months post mitral valve repair. Normal volunteers $(n=51)$. Image Acquisition: All participants underwent MRI on a $1.5 \mathrm{~T}$ scanner (GE Healthcare) optimized for cardiac application. An ECG-gated, breath-hold steady state free precision technique was used to obtain 2 chamber and 4 chamber views with 20 phase imaged in each cardiac cycle. Image Analysis: At end diastole (ED) and end systole (ES), anterior and inferior MA landmarks were manually marked on 2 chamber views. Septal, lateral and apex MA landmarks were marked on 4 chamber views. All landmarks were then automatically propagated to the remaining phases using custom software [1] and manually verified. In each phase, the MA was approximated by a flat plane fit to the 4 landmarks. The MA centroid and plane normal was
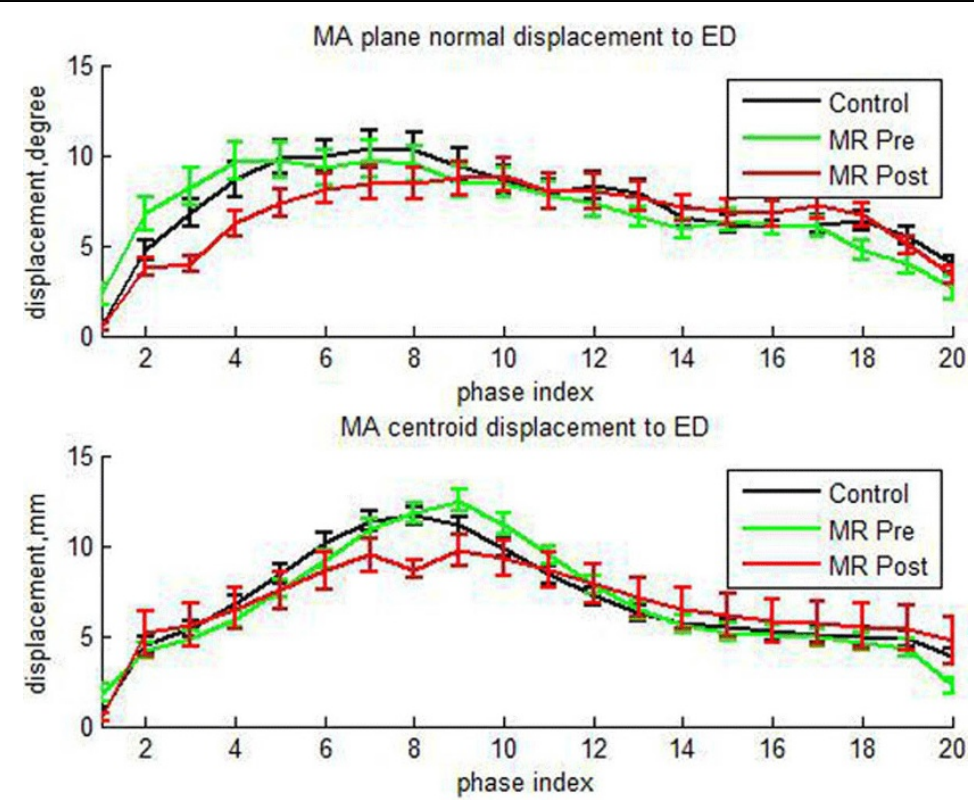

Figure 1 Mean \pm SE MA plane normal and centroid displacement to ED versus phase index.

${ }^{1} \mathrm{AU}$ MRI Research Center, Auburn University, Auburn, Alabama, USA

Full list of author information is available at the end of the article

(c) 2014 Zhang et al.; licensee BioMed Central Ltd. This is an Open Access article distributed under the terms of the Creative Commons 
Table 1 MRI-derived MV plane measurements

\begin{tabular}{|c|c|c|c|}
\hline & \multirow{2}{*}{$\begin{array}{l}\text { Control } \\
(n=51)\end{array}$} & \multicolumn{2}{|c|}{ MR } \\
\hline & & Preoperative $(n=35)$ & Postoperative $(n=35)$ \\
\hline MA Transverse Diameter at ED, mm & $33.07 \pm 5.52$ & $42.41 \pm 7.33^{*}$ & $31.61 \pm 3.88 \dagger$ \\
\hline MA Transverse Diameter at ES, mm & $35.95 \pm 4.07$ & $45.83 \pm 6.68^{*}$ & $32.26 \pm 5.1^{*} \dagger$ \\
\hline Septolateral Diameter at ED, mm & $35.07 \pm 3.74$ & $43.73 \pm 6.66^{*}$ & $34.51 \pm 5.99+$ \\
\hline Septolateral Diameter at ES, mm & $35.51 \pm 3.38$ & $43.58 \pm 7.52^{*}$ & $35.01 \pm 5.27 \dagger$ \\
\hline C. to A. distance at ED, $\mathrm{mm}$ & $95.40 \pm 7.75$ & $100.75 \pm 8.95^{*}$ & $96.65 \pm 9.21$ \\
\hline C. to A. distance at ES, mm & $79.94 \pm 7.49$ & $85.27 \pm 8.66^{*}$ & $84.66 \pm 8.02^{*}$ \\
\hline Left Ventricle Ejection Fraction,\% & $64 \pm 7$ & $61 \pm 7^{*}$ & $54 \pm 8^{*} \dagger$ \\
\hline Peak MA Anterior Displacement, mm & $10.53 \pm 3.09$ & $11.71 \pm 3.71$ & $8.16 \pm 2.8^{*} \dagger$ \\
\hline Peak E. Dia MA Anterior velocity, \% LA length/s & $64.02 \pm 25.1$ & $76.74 \pm 23.63^{*}$ & $55.19 \pm 19.64 \dagger$ \\
\hline Peak MA Inferior Displacement, mm & $12.73 \pm 3.94$ & $12.85 \pm 3.52$ & $8.6 \pm 2.43^{*} \dagger$ \\
\hline Peak E. Dia MA Inferior velocity, \% LA length/s & $64.93 \pm 35.6$ & $58.82 \pm 21.97$ & $39.83 \pm 18.47^{*} \dagger$ \\
\hline Peak MA Lateral Displacement, $\mathrm{mm}$ & $13.38 \pm 3.23$ & $13.91 \pm 3.09$ & $10.39 \pm 3.61^{*}+$ \\
\hline Peak E. Dia MA Lateral velocity, \% LA length/s & $79.32 \pm 34.3$ & $78.45 \pm 24.73$ & $49.4 \pm 16.36^{*}+$ \\
\hline Peak MA Septal Displacement, mm & $10.59 \pm 3.55$ & $12.04 \pm 3.1$ & $7.67 \pm 2.44^{*} \dagger$ \\
\hline Peak E. Dia MA Septal velocity, \% LA length/s & $71.26 \pm 27.44$ & $77.18 \pm 39.92$ & $43.03 \pm 16.9^{*}+$ \\
\hline Peak C. to A. dis. Displacement to pre., mm & $4.004 \pm 1.46$ & $3.759 \pm 1.35$ & $2.714 \pm 0.85^{*}+$ \\
\hline Peak normal displacement to ED, degree & $14.65 \pm 6.68$ & $14.46 \pm 5.53$ & $13.59 \pm 5.71$ \\
\hline
\end{tabular}

Values are mean \pm SE. MR, mitral regurgitation patients; ED: end diastole; ES: end systole; E Dia., early diastole; MA, mitral annulus; C., centroid; A., apex; dis., distance; pre., previous; LA, long axis; *, $\mathrm{P}<0.05$ vs. Control; $\dagger, \mathrm{P}<0.05$ vs. Preoperative.

computed in each plane. Differences in centroid position and plane normal angle were computed in each phase relative to ED.

\section{Results}

Centroid displacement and plane normal angle curves are shown in Figure 1 and parameters of these curves are shown in Table 1.

\section{Conclusions}

MR mitral annulus transverse diameter and septolateral diameter were significantly dilated in both ED and ES at baseline vs. controls, but were returned to normal level after surgery. However, we found that mitral annular mechanics in MR patients were impaired at 1 year postsurgery, demonstrated by the significant decrease in peak mitral annulus anterior, inferior, lateral and septal displacements as well as the peak early diastolic mitral annulus velocities normalized to the LVED length vs. controls.

\section{Funding}

\section{NIH/NHLBI P50-HL077100, R01 HL104018.}

\section{Authors' details}

'AU MRI Research Center, Auburn University, Auburn, Alabama, USA.

${ }^{2}$ Electrical and Computer Engineering Department, Auburn University,

Auburn, Alabama, USA. ${ }^{3}$ Department of Medicine, Division of Cardiovascular
Disease, University of Alabama at Birmingham, Birmingham, Alabama, USA. ${ }^{4}$ VA Medical Center, Birmingham, Alabama, USA.

Published: 16 January 2014

doi:10.1186/1532-429X-16-S1-P269

Cite this article as: Zhang et al:: Mitral annular kinetics in mitral regurgitation. Journal of Cardiovascular Magnetic Resonance 2014 16(Suppl 1):P269.

\section{Submit your next manuscript to BioMed Central and take full advantage of:}

- Convenient online submission

- Thorough peer review

- No space constraints or color figure charges

- Immediate publication on acceptance

- Inclusion in PubMed, CAS, Scopus and Google Scholar

- Research which is freely available for redistribution

Submit your manuscript at www.biomedcentral.com/submit
C Biomed Central 